Studies of bacteria on

leaf-cutting ants could

yield new antibiotics.

Other researchers call Currie's findings interesting, but say they wanted to see a more thorough analysis of the data. "It's interesting that he found these fungal enzymes in the gardens that he didn't expect [based on] what the fungus was capable of doing by itself," says John Taylor, a mycologist at the University of California, Berkeley.

Taylor says that Currie's continued scrutiny of the lives of ants provides insights into the web of interactions necessary for the survival of any single species. "I think the coolest thing about this is that you start with one organism, and then you find more and more organisms involved in the relationship," he says. It may take a village to raise a child; it seems it also takes a village to break down cellulose. Erika Check Hayden

\section{Visit http://tinyurl.com/ddh803 to see Cameron} Currie discuss his research.

April, Buerstedde expects to return to work.

Buerstedde admits that he can be impatient, but says that many enjoy working with him. One colleague told Nature that Buerstedde needed to learn to hold his tongue, but that he was appalled at the peremptory dismissal. "I didn't think it was possible in a country like Germany that someone could be dismissed without being given a chance to hear the charges or defend himself against them," said the colleague, who did not wish to be identified.

Some of Buerstedde's external collaborators have also expressed dismay in open letters. "I was truly shocked that you... have been fired so abruptly," wrote David Schatz, an immunologist at Yale University in New Haven, Connecticut. "It is a blow to the integrity of the research process and to academic freedom."

Alison Abbott

\title{
Quark statistics shed light on Universe's symmetry
}

The fundamental asymmetry in the laws of physics called charge-parity (CP) violation is tiny, yet it looms large enough in physics to have led to Nobel prizes on three occasions. A persistent puzzle is why the asymmetry is so small - some theories imply that it could, and perhaps should, be much bigger. Now, research ${ }^{1,2}$ is bolstering a previous suggestion that the smallness is not a mystery, but rather an inevitable consequence of another basic fact in physics: that the three known families of quarks have the masses that they do.

The findings, by Gary Gibbons and his colleagues at the University of Cambridge, UK, are spurring discussions about whether the laws of physics are 'fine-tuned' - that is, whether the magnitudes of various physical constants should be considered peculiarly unlikely. And they hint at the possibility of probing physics beyond the standard model, which describes all the known particles and forces at the subatomic scale.

$\mathrm{CP}$ violation means that some physical laws are altered if a subatomic particle is exchanged for its antiparticle, and at the same time left and right are swapped as in a mirror. The very subtle effect was first observed in 1964 in the way that exotic particles called neutral kaons decay. But some theories suggest that the asymmetry should be about a thousand times bigger than it is, leading scientists to wonder whether some unknown physical principle keeps the effect so small. Gibbons and his colleagues now suggest that the magnitude of the CP violation is just what should be expected given the observed masses of quarks (which make up protons, neutrons and other weighty particles).

The link between CP violation and quark mass is well known. In work that won them last year's physics Nobel, Japanese physicists Makoto Kobayashi and Toshihide Maskawa showed in 1973 that CP violations are inevitable if there are more than two types of quark (and corresponding antiquarks) that have different masses. Their finding connected CP violation to the hierarchy of quark masses, and suggested a way to work out its magnitude.

That shifted the question to why quark families have the masses they do something not explained by the standard model, but that could fall out of a deeper, as yet unknown theory. In 1998, John Donoghue of the University of Massachusetts in Amherst suggested ${ }^{3}$ that rather than predicting exact masses for specific quarks, such a theory might predict a 'landscape' of allowed masses, of which those observed in this Universe are typical examples.

In a 2006 paper $^{4}$, he and his co-workers showed that, by assuming a simple distribution of possible values for the quark masses, they could calculate the range of the most likely values of a parameter called $J$ that quantifies CP violation. And they found the observed value fell squarely within that range. Gibbons and his colleagues now find much the same result when they assume a different statistical distribution of quark masses in the hypothetical landscape. "I can only speculate why the earlier work went largely unseen," says Max Tegmark of the Massachusetts Institute of Technology in Cambridge.

Tegmark says the work is "very interesting", but others disagree about its significance. Physicist Alexei Grinbaum of CEA-Saclay in Gif-sur-Yvette, France, says that the results do not dismiss the fine-tuning issue, but just shift the responsibility for fine-tuning to the hierarchy of quark masses. Particle physicist Graham Ross of the University of Oxford, UK, agrees with that, but also feels that the mass hierarchy can itself by explained by symmetry arguments - so there is no great mystery in the first place.

Philip Ball

1. Gibbons, G. W., Gielen, S., Pope, C. N. \& Turok, N. Phys. Rev. Lett. 102, 121802 (2009).

2. Gibbons, G. W., Gielen, S., Pope, C. N. \& Turok, N. Phys Rev. D 79, 013009 (2009).

3. Donoghue, J. F. Phys. Rev. D 57, 5499-5508 (1998)

4. Donoghue, J. F., Dutta, K. \& Ross, A. Phys. Rev. D 73 113002 (2006). 